\title{
MONOTONE AND COMONOTONE APPROXIMATION
}

\author{
E. PASSOW AND L. RAYMON
}

ABSTRACT. Jackson type theorems are obtained for monotone and comonotone approximation. Namely

(i) If $f(x)$ is a function such that the $k$ th difference of $f$ is $\geqq 0$ on $[a, b]$ then the degree of approximation of $f$ by $n$th degree polynomials with $k$ th derivative $\geqq 0$ is $0\left[\omega\left(f ; 1 / n^{1-\varepsilon}\right)\right]$ for any $\varepsilon>0$, where $\omega(f ; \delta)$ is the modulus of continuity of $f$ on $[a, b]$.

(ii) If $f(x)$ is piecewise monotone on $[a, b]$ then the degree of approximation of $f$ by $n$th degree polynomials cononotone with $f$ is $0\left[\omega\left(f ; 1 / n^{1-\varepsilon}\right)\right]$ for any $\varepsilon>0$.

The degree of approximation of a real function $f \in C[a, b]$ by a space of functions $\mathscr{P}$ is

$$
E(f ; \mathscr{P})=\inf _{P \in \mathscr{P}}\|f-P\|,
$$

where the norm is the ordinary sup norm. Jackson's classic theorem states that the degree of approximation of a function $f \in C[a, b]$ by the space $\mathscr{P}_{n}$ of algebraic polynomials of degree $\leqq n$ satisfies

$$
E\left(f ; \mathscr{P}_{n}\right)=E_{n}(f) \leqq C \omega(f ; 1 / n),
$$

where $C>0$ is a constant not depending on $n$ or $f$, and $\omega(f ; \delta)$ is the modulus of continuity of $f$. It is natural to ask to what extent the degree of approximation to $f$ is affected by replacing the space of approximating functions $\mathscr{P}_{n}$ by another (restricted) space $\mathscr{P}_{n}^{*} \subset \mathscr{P}_{n}$. In this article we address ourselves to two related questions of this type, (A) monotone approximation and (B) comonotone approximation.

(A) Monotone approximation. How closely can one approximate a monotone function $f$ on $[a, b]$ by a polynomial that is monotone on $[a, b]$ ? I.e., what is the degree of approximation of $f$ by the space of polynomials of degree $\leqq n$ that are monotone on $[a, b]$ ? More generally, if $\mathscr{P}_{n . k}$ denotes the space of polynomials $P$ of degree $\leqq n$ satisfying $P^{(k)}(x) \geqq 0$ on $[a, b]$, then what is the degree of approximation $E\left(f ; \mathscr{P}_{n, k}\right)=E_{n, k}(f)$ where $f(x)$ is a function whose $k$ th difference $\Delta^{k} f$ is always $\geqq 0$ on $[a, b]$ ?

Received by the editors February 26, 1973.

AMS (MOS) subject classifications (1970). Primary 41A25, 41A10, $42 \mathrm{~A} 08$.

Key words and phrases. Monotone approximation, comonotone approximation, piecewise monotone approximation, Jackson theorem. 
These questions were first raised by Shisha in [5], where he proved that if $f^{(k)}(x) \geqq 0$ and $f^{(p)}(x) \in \operatorname{Lip} 1$, where $1 \leqq k \leqq p$, then

$$
\begin{aligned}
E_{n . k}(f) & \leqq C(\pi / 4)^{p-k+1}(b-a)^{p+1}\left[k ! \prod_{j=k}^{p}(n+1-j)\right]^{-1} \\
& \leqq \frac{C_{p . k}}{(n-p)^{p-k+1}} .
\end{aligned}
$$

Roulier [4] has obtained results that represent some improvement over Shisha's in certain cases where $k=p \geqq 2$. If $f$ is not assumed to be in $C^{p}$ for any $p \geqq 1$, the question of an estimate on the order of magnitude of $E_{n, k}$ remains. For the case $k=1$, Lorentz and Zeller [1] have obtained a very satisfying result. They have shown that for a monotone function $f$

$$
E_{n, 1}(f)=O[\omega(f ; 1 / n)] .
$$

This is the same order of magnitude as that given by Jackson's theorem for "unrestricted" approximation (1). In Theorem 3 we are able to show that if the $k$ th difference $\Delta^{k} f$ of $f$ is $\geqq 0$, then for every $\varepsilon>0$

$$
E_{n, k}(f)=o\left[\omega\left(f ; 1 / n^{1-\varepsilon}\right)\right] .
$$

(B) Comonotone approximation. $f$ will be called piecewise monotone if it has only a finite number of local maxima and minima in $[a, b]$. The local maxima and minima of $f$ in $[a, b]$ together with the endpoints $a, b$ will be referred to as the peaks of $f$. If $g$ is nondecreasing on the subintervals of $[a, b]$ on which $f$ is nondecreasing, and nonincreasing on those subintervals on which $f$ is nonincreasing, then $g$ is said to be comonotone with $f$. Given a piecewise monotone function $f(x)$ let $\mathscr{P}_{n}^{*}(f)$ denote the space of all polynomials of degree $\leqq n$ that are comonotone with $f$ on $[a, b]$; let $E_{n}^{*}(f)$ denote the comonotone degree of approximation of $f$; i.e.,

$$
E_{n}^{*}(f)=E_{n}\left[f ; \mathscr{P}_{n}^{*}(f)\right] .
$$

By Jackson's theorem $E_{n}(f)=O[\omega(f ; 1 / n)]$. What is the order of magnitude of $E_{n}^{*}(f)$ ? Newman, Passow and Raymon [2] have obtained results of a modified nature. They have shown that for $n$ sufficiently large there is $P \in \mathscr{P}_{n}$ such that $\|f-P\|<C \omega(f ; 1 / n)$ where $f$ and $P$ are comonotone except in certain neighborhoods (whose diameters tend to zero with $n$ ) of the peaks. Also, Passow and Raymon have obtained an estimate for "perfectly" comonotone approximation for functions in $C^{p}$ [3]: If $f(x)$ has $k$ peaks and $f \in C^{p}[a, b]$ with $p>k$ and with $f^{(p)} \in \operatorname{Lip} 1[a, b]$, then

$$
E_{n}^{*}(f) \leqq(b-a)^{k+1}(C / n)^{p-k-1}
$$

whenever $n>2 p$, where $C$ is independent of $n, p, f$ and $k$. 
Let $S$ be a set of functions. We shall use the following notation:

$$
E_{n}^{*}(S)=\sup _{f \in S} E_{n}^{*}(f)
$$

Theorem 1 relates the comonotone degree of approximation $E_{n}^{*}(f)$ of an arbitrary function $f$ to $E_{n}^{*}(S)$ where $S$ is a class of functions satisfying certain smoothness conditions. Theorem 2 is proved easily from (3) and Theorem 1.

THEOREM 1. Let $S^{p}$ denote the set of functions $g$ in $C^{p}[a, b]$ such that $g^{(p)}$ is a contraction on $[a, b]$ (i.e., $\omega\left(g^{(p)} ; \delta\right) \leqq \delta$ for all $\left.\delta>0\right)$. Let $a=x_{0}<x_{1}<\cdots<x_{k}=b$ be the peaks of a piecewise monotone function $f(x)$ on $[a, b] ;$ let $\delta=\frac{1}{2} \min _{1 \leqq i \leqq k}\left|x_{i}-x_{i-1}\right|$. Let $\lambda=\lambda_{n}=\left[E_{n}^{*}\left(S^{D}\right)\right]^{1 /(p+1)}$. Then

whenever $p \lambda_{n}<\delta$.

$$
E_{n}^{*}(f) \leqq p^{2} 2^{p+1} \omega\left(f ; \lambda_{n}\right)
$$

ProOF. Let $f^{*}(x)$ be defined on $[a, b+p \lambda]$ as follows:

$$
\begin{aligned}
f^{*}(x) & =f\left(x_{i}\right), & & x_{i} \leqq x \leqq x_{i}+p \lambda, i=1,2, \cdots, k, \\
& =f(x), & & \text { for all other } x .
\end{aligned}
$$

$f^{*}(x)$ is comonotone with $f(x)$ on $[a, b]$. In addition, the monotonicity of $f^{*}(x)$ on $\left[x_{i-1}, x_{i}\right]$ extends to $\left[x_{i-1}, x_{i}+p \lambda\right], i=1,2, \cdots, k$. From the definition of $f^{*}(x)$ and by the sublinear property of the modulus of continuity we deduce

$$
\omega\left(f^{*} ; \lambda\right) \leqq \omega(f ; p \lambda) \leqq p \omega(f ; \lambda)
$$

and

$$
\left\|f-f^{*}\right\| \leqq \omega(f ; p \lambda) \leqq p \omega(f ; \lambda)
$$

Let

$$
g(x)=\frac{1}{\lambda^{p+1}} \int_{x}^{x+\lambda} \int_{t_{\mathcal{D}}}^{t_{\mathcal{D}}+\lambda} \int_{t_{\mathcal{D}-1}}^{t_{\mathcal{D}-1+\lambda}} \cdots \int_{t_{1}}^{t_{1}+\lambda} f^{*}(t) d t d t_{1} d t_{2} \cdots d t_{p} .
$$

We shall show that $f$ and $g$ are comonotone. If $f(x)$ is nondecreasing on $\left[x_{i-1}, x_{i}\right]$, then $f^{*}(x)$ is nondecreasing on $\left[x_{i-1}, x_{i}+p \lambda\right]$ and $g_{1}(x)=\int_{x}^{x+\lambda} f^{*}(t) d t$ is nondecreasing on $\left[x_{i-1}, x_{i}+(p-1) \lambda\right] ; g_{2}(x)=$ $\int_{x}^{x+\lambda} \int_{t_{1}}^{t_{1}+\lambda} f^{*}(t) d t d t_{1}$ is nondecreasing on $\left[x_{i-1}, x_{i}+(p-2) \lambda\right]$; Iterating the procedure $p$ times, we conclude that $g(x)$ is nondecreasing on $\left[x_{i-1}, x_{i}\right]$. Similarly, if $f(x)$ is nonincreasing on $\left[x_{i-1}, x_{i}\right]$, then $g(x)$ is nonincreasing on the same interval, and $g(x)$ is comonotone with $f(x)$.

Applying the Fundamental Theorem of Calculus $p$ times to $g(x)$ we 
conclude that

$$
g^{(p)}(x)=\frac{1}{\lambda^{p+1}} \sum_{j=0}^{p}(-1)^{j}\left(\begin{array}{l}
p \\
j
\end{array}\right) \int_{x+(p-j) \lambda}^{x+(p-j+1) \lambda} f^{*}(t) d t .
$$

Then, since $f^{*}$ is continuous except at a finite number of points,

$$
\left|g^{(p+1)}(x)\right| \leqq \frac{2^{p}}{\lambda^{p+1}} \omega\left(f^{*}, \lambda\right) \leqq \frac{p 2^{p}}{\lambda^{p+1}} \omega(f ; \lambda), \quad \text { by (4). }
$$

Hence $\lambda^{p+1} g(x) / p 2^{p} \omega(f ; \lambda) \in S^{p}$. Then there is some polynomial $Q(x) \in$ $\mathscr{P}_{n}^{*}(f)$ such that

$$
\left\|\lambda^{p+1} g(x) / p 2^{p} \omega(f ; \lambda)-Q(x)\right\| \leqq E_{n}^{*}\left(S^{p}\right)=\lambda^{p+1} .
$$

Then, if $P(x)=p 2^{p} \omega(f ; \lambda) Q(x) / \lambda^{p+1}, P \in \mathscr{P}_{n}^{*}(f)$ and

$$
\|g(x)-P(x)\| \leqq p 2^{p} \omega(f ; \lambda)
$$

Also

$$
\begin{aligned}
\left\|g-f^{*}\right\| & =\left\|\frac{1}{\lambda^{p+1}} \int_{x}^{x+\lambda} \int_{t_{D}}^{t_{p}+\lambda} \cdots \int_{t_{1}}^{t_{1}+\lambda}\left[f^{*}(t)-f^{*}(x)\right] d t d t_{1} \cdots d t_{p}\right\| \\
& \leqq \frac{\omega\left(f^{*} ; p \lambda\right)}{\lambda^{p+1}}\left\|\int_{x}^{x+\lambda} \int_{t_{D}}^{t_{p}+\lambda} \cdots \int_{t_{1}}^{t_{1}+\lambda} d t d t_{1} \cdots d t_{\nu}\right\| \\
& \leqq \omega\left(f ; p^{2} \lambda\right) \leqq p^{2} \omega(f ; \lambda) .
\end{aligned}
$$

Now, from (5), (6) and (7), we have

$$
\begin{aligned}
E_{n}^{*}(f) & \leqq\|f-P\| \leqq\left\|f-f^{*}\right\|+\left\|f^{*}-g\right\|+\|g-P\| \\
& \leqq\left(p+p^{2}+p 2^{p}\right) \omega(f ; \lambda) \leqq p^{2} 2^{p+1} \omega(f ; \lambda),
\end{aligned}
$$

and the proof is complete.

THEOREM 2. If $f(x)$ is a piecewise monotone function on $[a, b]$, then for any $\varepsilon>0$ there is some constant $b_{k, \varepsilon}>0$ such that for $n$ sufficiently large $E_{n}^{*}(f) \leqq b_{k, \varepsilon} \omega\left(f ; 1 / n^{1-\varepsilon}\right)$; i.e.,

$$
E_{n}^{*}(f)=o\left[\omega\left(f ; 1 / n^{1-\varepsilon}\right)\right] .
$$

- Proof. Suppose $f(x)$ has $k$ peaks. By (3), $E_{n}^{*}\left(S^{p}\right) \leqq(b-a)^{k+1}(C / n)^{p-k-1}$ whenever $n>2 p$. If $p$ is chosen so large that $(p-k-1) /(p+1)>1-\varepsilon$, then $\lambda_{n}=\left[E_{n}^{*}\left(S^{p}\right)\right]^{1 /(p+1)}=o\left(1 / n^{1-\varepsilon}\right)$, and Theorem 2 then follows from Theorem 1.

THEOREM 3. If $f(x)$ is a function such that for all $x$ the kth difference $\Delta^{k} f(x) \geqq 0$ on $[a, b]$, then for any $\varepsilon>0$ there is some constant $d_{k, \varepsilon}>0$ such that for $n$ sufficiently large

$$
E_{n . k}(f) \leqq d_{k, \varepsilon} \omega\left(f ; 1 / n^{1-\varepsilon}\right) ; \text { i.e., } \quad E_{n, k}(f)=o\left[\omega\left(f ; 1 / n^{1-\varepsilon}\right)\right] .
$$


Proof. Let

$$
g(x)=1 / \lambda^{p+k+1} \int_{x}^{x+\lambda} \int_{t_{p+k}}^{t_{p+k+\lambda}} \int_{t_{p+k-1}}^{t_{p+k-1}+\lambda} \cdots \int_{t_{1}}^{t_{1}+\lambda} f(t) d t d t_{1} \cdots d t_{p+k},
$$

where $p$ and $\lambda>0$ will be specified later. Then

$$
\|g-f\| \leqq \omega[f ;(p+k+1) \lambda] \leqq(p+k+1) \omega(f ; \lambda) .
$$

Applying the Fundamental Theorem of Calculus to $g(x) k$ times,

$$
g^{(k)}(x)=\frac{1}{\lambda^{p+1}} \int_{x}^{x+\lambda} \int_{t_{p}}^{t_{p}+\lambda} \cdots \int_{t_{1}}^{t_{1}+\lambda} \frac{\Delta_{\lambda}^{k} f(t)}{\lambda^{k}} d t d t_{1} \cdots d t_{p}
$$

Since the integrand in (9) is assumed nonnegative, $g^{(k)}(x) \geqq 0$. Moreover, $\left|g^{(p+k+1)}(x)\right| \leqq 2^{p+k} \omega(f ; \lambda) / \lambda^{p+k+1}$. Hence if $h(x)=\lambda^{p+k+1} g(x) / 2^{p+k} \omega(f ; \lambda)$, then $h(x) \in S^{p+k}$; then by Shisha's theorem (2) there is a polynomial $Q(x) \in \mathscr{P}_{n, k}$ such that

$$
E_{n, k}(h) \leqq\|h-Q\| \leqq C_{p, k} /(n-p)^{p+1} .
$$

Therefore, letting $P(x)=2^{p+k} \omega(f ; \lambda) Q(x) / \lambda^{p+k+1}, P \in \mathscr{P}_{n . k}$ and

$$
\|g-P\| \leqq C_{p, k} 2^{p+k} \omega(f ; \lambda) / \lambda^{p+k+1}(n-p)^{p+1} .
$$

Hence, by (8) and (10),

$$
\begin{aligned}
\|f-P\| & \leqq\|f-g\|+\|g-P\| \\
& <\omega(f ; \lambda)\left[p+k+1+C_{p, k} 2^{p+k} /(n-p)^{p+1} \lambda^{p+k+1}\right] .
\end{aligned}
$$

Choose $p$ so large that $(p+1) /(p+k+1)>1-\varepsilon$, and then choose $\lambda=(n-p)^{-(p+1) /(p+k+1)}$. Then

$$
\|f-P\| \leqq d_{k, \varepsilon} \omega\left(f ; 1 / n^{1-\varepsilon}\right) \text { and } \quad E_{n, k}(f)=o\left[\omega\left(f ; 1 / n^{1-\varepsilon}\right)\right] . \quad \text { Q.E.D. }
$$

\section{REFERENCES}

1. G. G. Lorentz and K. L. Zeller, Degree of approximation by monotone polynomials. I, J. Approximation Theory 1 (1968), 501-504. MR 39 \#699.

2. D. J. Newman, E. Passow and L. Raymon, Piecewise monotone polynomial approximation, Trans. Amer. Math. Soc. 172 (1972), 465-472.

3. E. Passow and L. Raymon, Comonotone polynomial approximation, J. Approximation Theory (to appear).

4. J. A. Roulier, Monotone approximation of certain classes of functions, J. Approximation Theory 1 (1968), 319-324. MR 38 \#4875.

5. O. Shisha, Monotone approximation, Pacific J. Math. 15 (1965), 667-671. MR 32 \#2802.

Department of Mathematics, Temple University, Philadelphia, Pennsylvania 19122 